\title{
Objective vs Subjective Vault Measurement After Myopic Implantable Collamer Lens Implantation
}

\author{
JOSÉ F. ALFONSO, CARLOS LISA, ANA PALACIOS, PAULO FERNANDES, JOSÉ M. GONZÁlEZ-MÉIJOME, \\ AND ROBERT MONTÉS-MICÓ
}

- PURPOSE: To evaluate the relationship between subjective measurements of vault and objective values measured with Visante optical coherence tomography (OCT) in eyes receiving an implantable contact lens (ICL) for myopia correction.

- DESIGN: Observational cross-sectional study.

- METHODS: SETTING: Fernández-Vega Ophthalmological Institute, Oviedo, Spain. PATIENTs: Four hundred and fifty-two eyes from 246 patients were elected to be implanted with a Visian ICL V4 (STAAR Surgical Inc, Monrovia, California, USA). OBSERVATION PROCEDURES: Subjective and objective measurements of vault after implantation of ICL. MAIN OUTCOME MEASURES: Subjective vault classified in 5 levels assessed using an optical section during slit-lamp examination. Objective vault was measured with Visante OCT (Carl Zeiss Meditec Inc, Dublin, California, USA).

- RESULTS: Average values of objective and subjective vault were $414 \pm 228 \mu \mathrm{m}$ and $2.1 \pm 1.0$, respectively and both parameters were highly correlated $(r=0.82$; $P<.001)$. Differences in average objective vault were statistically significant among the 5 groups of subjective vault $(P<.001)$. Subjective vault 0 corresponded to a mean OCT value of $62 \pm 49 \mu \mathrm{m}$ with $99 \%$ confidence interval (CI) $[38 ; 86] \mu \mathrm{m}$; eyes with vault 1 to $203 \pm 93$ $\mu \mathrm{m}$ with $99 \%$ CI $[176 ; 230] \mu \mathrm{m}$; eyes with vault 2 to $402 \pm 131 \mu \mathrm{m}$ with $99 \%$ CI $[378 ; 425] \mu \mathrm{m}$; eyes with vault 3 to $594 \pm 146 \mu \mathrm{m}, 99 \%$ CI $[554 ; 633] \mu \mathrm{m}$; and vault 4 to $794 \pm 182 \mu \mathrm{m}$ with $99 \% \mathrm{CI}[713 ; 875] \mu \mathrm{m}$. - CONCLUSIONS: Subjective and objective values of vault are highly correlated. In $99 \%$ of cases within the CI, objective values for eyes subjectively classified within a certain level vary within a narrow interval $( \pm 25$ to 80 $\mu \mathrm{m})$ around the mean value, and this interval is characteristic of each subjective level. (Am J Ophthalmol

Accepted for publication Jan 12, 2009.

From the Fernández-Vega Ophthalmological Institute (J.F.A., C.L., A.P.); the Surgery Department, School of Medicine, University of Oviedo, Oviedo, Spain (J.F.A.); the Department of Physics (Optometry), School of Sciences, University of Minho, Braga, Portugal (P.F., J.M.G.M.); and the Optics Department, Faculty of Physics, University of Valencia, Valencia, Spain (R.M.-M.).

Inquiries to José F. Alfonso, Instituto Oftalmológico Fernández-Vega, Avda. Dres. Fernández-Vega 114, 33012 Oviedo, Spain; e-mail: j.alfonso@fernandez-vega.com
2009;147:978-983. (C) 2009 by Elsevier Inc. All rights reserved.)

MPLANTATION OF POSTERIOR CHAMBER (PC) PHAKIC intraocular lens (IOL) has been increasingly performed to correct ametropia, ${ }^{1-4}$ particularly of moderate and high degree because of concerns with predictability, optical quality of the eye, and different complications related with ablation procedures such as photorefractive keratectomy (PRK) or laser in situ keratomileusis (LASIK). Also, in several corneal conditions that contraindicate photorefractive procedures, such as keratoconus, ${ }^{5}$ PC phakic IOL implantation may be a feasible option to correct or reduce associated refractive errors.

Visian IOL (STAAR Surgical Inc, Monrovia, California, USA), also known as implantable collamer lens (ICL), is a phakic IOL designed to be placed in the PC behind the iris with the haptic zone resting on the ciliary sulcus, and an anterior vault designed to avoid contact with the anterior surface of the crystalline lens. Despite that overall good refractive outcomes can be achieved with these lenses in terms of predictability, efficacy, safety, and stability over time, ${ }^{2,6,7}$ several short-term and long-term potential complications have been reported, including cataractogenesis, pigment dispersion, peripheral anterior synechia, or pupillary block glaucoma. ${ }^{8-11}$ Cataract formation is the major concern and may result from continuous contact between the IOL and the lens capsule or from direct surgical trauma. ${ }^{12-17}$ Physical distance between anterior surface of crystalline lens and the posterior surface of the ICL is defined as lens vault, and its clinical assessment is an important concern for safety regarding ICL implantation. In the clinical setting, vault assessment was first performed subjectively comparing this distance against the apparent corneal thickness from the optical section during slit-lamp examination or using Scheimpflug photography techniques. ${ }^{15,16,18,19}$ More recently, the physical separation between the lens and the ICL can be objectively measured using optical coherence tomography (OCT) with micrometric resolution. ${ }^{20-23}$

The purpose of this study was to evaluate the relationship between objective and subjective assessment of vault and to evaluate if subjective vault is a consistent measure of the separation between the ICL and the crystalline lens 
in a large population of patients receiving an ICL implantation for myopia correction.

\section{PATIENTS AND METHODS}

- STUDY DESIGN: Four-hundred and fifty-two myopic eyes from 246 patients who underwent a Visian ICL V4 (STAAR Surgical Inc) implantation (206 bilateral and 40 unilateral) were enrolled in this study. Mean patient age at the time of surgery was $32.64 \pm 6.05$ years (range, 18 to 50 years); 150 subjects $(61.0 \%)$ were female and $96(39.0 \%)$ were male.

Inclusion criteria required subjects to be myopic in 1 or both eyes and to be between 18 and 55 years of age. Exclusion criteria included history of glaucoma, retinal detachment, or other serious retinal degeneration related or not with myopia, corneal disease, previous corneal or intraocular surgery, abnormal iris, macular degeneration or retinopathy, neuro-ophthalmic disease, and history of ocular inflammation. Informed consent was obtained from all patients after the nature and possible consequences of the study were explained.

All surgeries were performed by 1 experienced surgeon (J.F.A.) at the department of refractive surgery of the Ophthalmologic Institute Fernández-Vega (Spain). All surgeries were performed through a 3.2-mm clear corneal tunnel incision in the steepest meridian using peribulbar anesthesia. Thirty minutes before surgery, cycloplegic and phenylephrine eye drops were instilled. Five minutes before surgery, povidone-iodine 5\% (Betadine; Meda Manufacturing, Bordeaux, France) was instilled. The anterior chamber $(\mathrm{AC})$ was filled with sodium hyaluronate $1 \%$ (Provisc; Alcon Laboratories Inc, Fort Worth, Texas, USA), which was completely removed at the end of the surgery. Tobramycin and dexamethasone $0.1 \%$ (Tobradex; Alcon Laboratories Inc) eye drops were used 4 times a day for 7 days, after which diclofenac sodium eye drops (Voltaren; Novartis Pharmaceuticals, Basel, Switzerland) were started 4 times a day for 2 weeks. Intraocular pressure was rigorously monitored. In cases of bilateral implantation, the second eye was operated within 1 week after the first surgery. The ICL power was calculated using the software ICL power table from STAAR Surgical Inc. Laser iridotomy was performed 1 week before the surgery.

- VAULT ASSESSMENT: Subjective and objective vault assessment was obtained at 1 of the follow-up visits between 3 and 6 months after surgery. Both subjective and objective values were obtained at the same visit, and subjective assessment was always done first. Objective assessment was carried out later by another trained member of the staff masked to the subjective value previously obtained.

Subjective vault classified in 5 levels was assessed by comparing the separation between the lens and the poste-
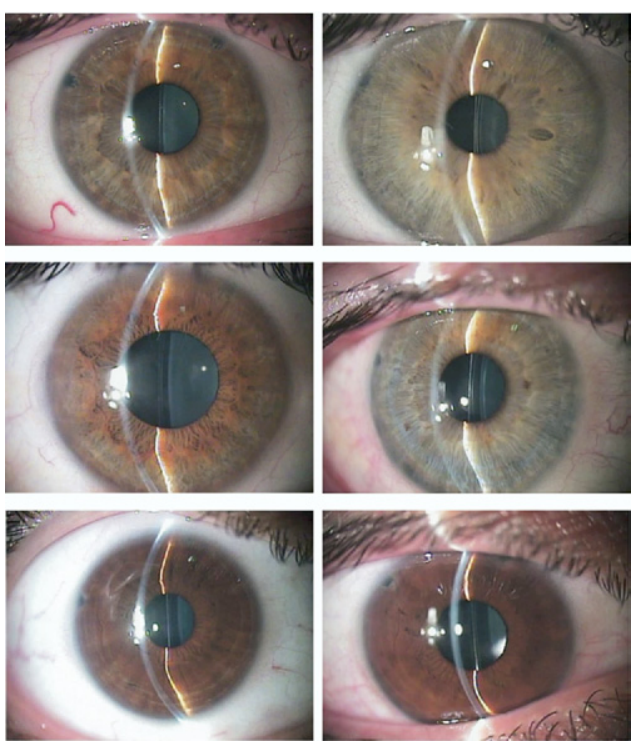

FIGURE 1. Clinical photographs showing different degrees of subjective vault from top left to bottom right: vault 0 , vault 1 , vault 2 , vault 3 , vault 4 , and vault $>4$.

TABLE 1. Descriptive Statistics for Demographic Data of Patients and Characteristics of Implanted Implantable Collamer Lens

\begin{tabular}{lrcl}
\hline & Mean & $\begin{array}{c}\text { Standard } \\
\text { Deviation }\end{array}$ & $\begin{array}{c}\text { Range } \\
\text { [Min, Max] }\end{array}$ \\
\hline Age (years) & 32.65 & 6.05 & {$[18,50]$} \\
Spherical refraction (D) & -9.17 & 3.85 & {$[-24,-1]$} \\
Astigmatism (D) & -1.27 & 1.04 & {$[-6,-1]$} \\
ICL size (mm) & 12.19 & 0.32 & {$[11.5,13.0]$} \\
ICL power (D) & -12.76 & 4.23 & {$[-23,-3]$} \\
White to white (mm) & 11.70 & 0.35 & {$[10.5,13.2]$} \\
ACD (mm) & 3.45 & 0.35 & {$[3.05,3.96]$}
\end{tabular}

$\mathrm{ACD}=$ anterior chamber depth; $\mathrm{D}=$ diopters; $\mathrm{ICL}=$ implantable collamer lens.

rior surface of the ICL to the corneal thickness using an optical section during routine slit-lamp examination. The following criteria are used to rate vault value: vault 0 , ICL apparently touches the anterior capsule of the lens; vault 1 , separation lower than half of corneal thickness; vault 2 , separation equal to corneal thickness; vault 3, separation larger than corneal thickness; or vault 4, separation about twice the corneal thickness. Examples of different degrees of vault are shown in Figure 1. This classification is slightly different from that recommended by the manufacturer. Considering our previous experience and the data we have now obtained with Visante OCT, the range of values commonly used in our patients is up to 1200 to $1400 \mu \mathrm{m}$ (which is about $2 \times$ corneal thickness). According to this, 
TABLE 2. Number of Eyes, Mean, Standard Deviation, 99\% Confidence Interval, and Range for Individual Value of Subjective Vault Readings and Optical Coherence Tomography Value $(\mu \mathrm{m})$ After Implantable Collamer Lens Implantation

\begin{tabular}{|c|c|c|c|c|c|c|c|}
\hline \multirow{2}{*}{$\begin{array}{c}\text { Subjective } \\
\text { Vault } \\
\text { Grade }\end{array}$} & \multirow[b]{2}{*}{ Eyes (\%) } & \multirow[b]{2}{*}{ Mean } & \multirow[b]{2}{*}{ SD } & \multicolumn{2}{|c|}{$\begin{array}{c}99 \% \\
\text { Confidence } \\
\text { Interval }\end{array}$} & \multirow[b]{2}{*}{ Minimum } & \multirow[b]{2}{*}{ Maximum } \\
\hline & & & & $\begin{array}{c}\text { Lower } \\
\text { Limit }\end{array}$ & $\begin{array}{l}\text { Upper } \\
\text { Limit }\end{array}$ & & \\
\hline 0 & $31(6.9)$ & 62 & 49 & 38 & 86 & 0 & 220 \\
\hline 1 & 83 (18.4) & 203 & 93 & 176 & 230 & 0 & 508 \\
\hline 2 & 206 (45.6) & 402 & 131 & 378 & 425 & 30 & 760 \\
\hline 3 & 95 (21) & 594 & 146 & 554 & 633 & 280 & 910 \\
\hline 4 & $37(8.1)$ & 794 & 182 & 713 & 875 & 400 & 1300 \\
\hline
\end{tabular}

$\mathrm{SD}=$ standard deviation

we were usually faced with vault values that did not cover the whole scale (up to 4). Rather than that, using the manufacturer's criteria, all our values will fall within half of the scale, taking values of 0,1 , and 2 . As a consequence, the clinical utility and sensitivity to detect small changes was very low. Considering each increase in vault as $1 \times$ corneal thickness, we will have a resolution of about 500 $\mu \mathrm{m}$, while considering our current strategy we will have a resolution of about half that value, or $250 \mu \mathrm{m}$.

Objective vault assessment was done using optical coherence tomography technology in the Visante OCT device (Carl Zeiss Meditec Inc, Dublin, California, USA). OCT uses an interferometric, noninvasive optical tomographic imaging technique that provides sharper images up to several millimeters of depth with sub-micrometer axial and lateral resolution. Vault between the lens and the ICL was measured perpendicular to the lens apex or at the narrowest space between both surfaces.

All subjective and objective vault measures were taken under the same light conditions and under cycloplegic effect in order to avoid potential influence of accommodation-induced changes in the position of the anterior surface of the crystalline lens or the ICL itself on the estimation of the vault value.

- DATA ANALYSIS: Statistical analysis was performed using SPSS statistical package version 16.0 (SPSS Inc, Chicago, Illinois, USA). Descriptive statistics were obtained. In order to evaluate normality of data distribution Kolmogorov-Smirnov test was used. Bivariate correlations were produced using parametric (Pearson's coefficient) or nonparametric (Spearman's coefficient) correlation analysis, depending on normal or non-normal distribution of variables. Kruskal-Wallis test was performed to explore statistical differences in values of objective vault for each subjective vault. The $99 \%$ confidence intervals (CI) of objective vault values as obtained with OCT were ob-

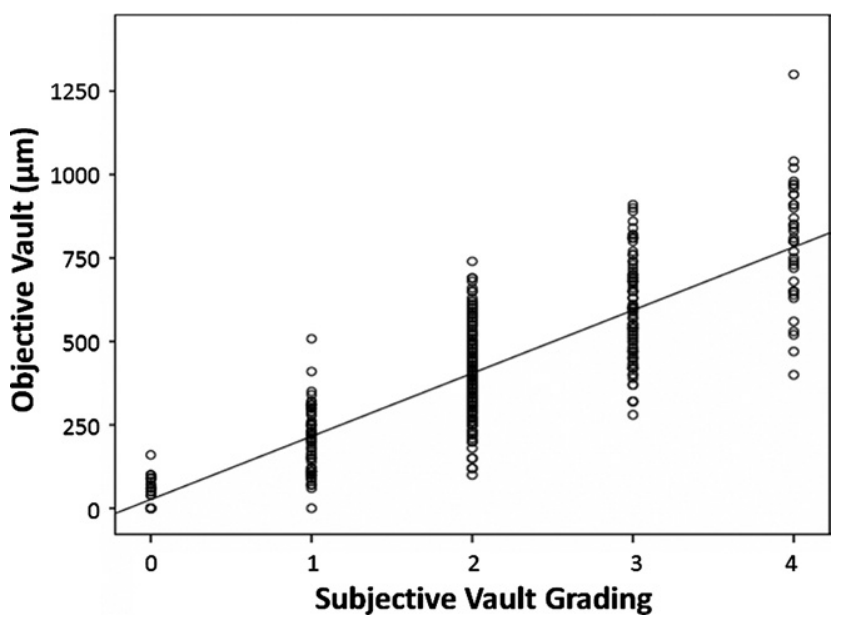

FIGURE 2. Scatterplot for the 5 levels of subjective vault grade (separation between the lens and the posterior surface of the implantable collamer lens [ICL] compared to the corneal thickness) vs objective vault (measured with optical coherence tomography) values. There is a statistically significant correlation between subjective and objective values $(r=0.82 ; P<$ .001) after ICL implantation.

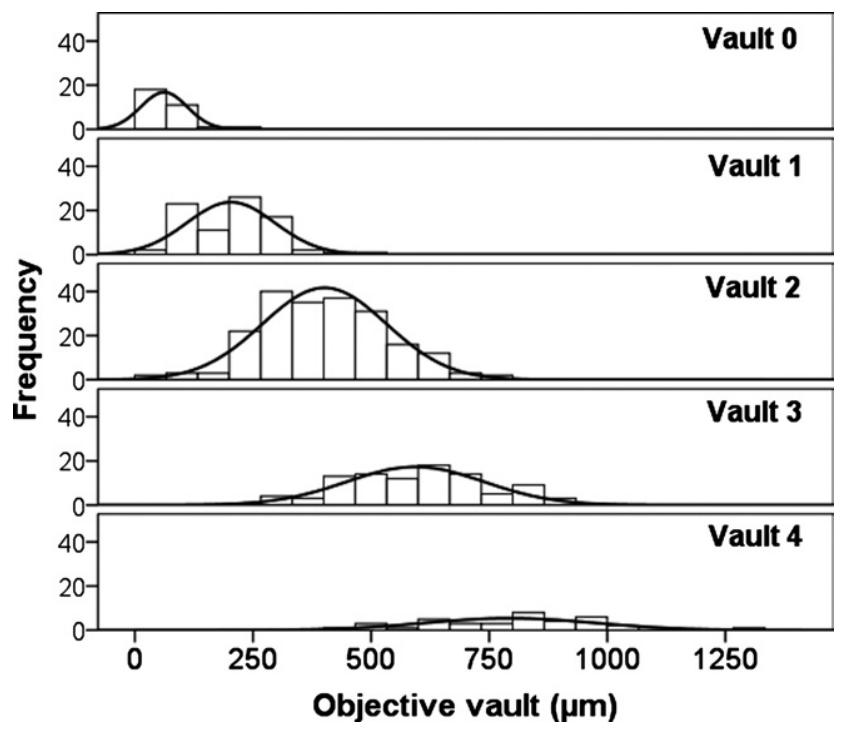

FIGURE 3. Frequency distribution of objective vault values among different subjective vault group after ICL implantation.

tained for each grade of subjective vault. Differences were considered statistically significant when the $P$ value was less than .05 .

\section{RESULTS}

THE MEAN MANIFEST SPHERICAL REFRACTION WAS $-9.17 \pm$ 3.85 diopters (D) (range, -1.00 to $-24.00 \mathrm{D}$ ), with a mean manifest astigmatism of $-1.27 \pm 1.04 \mathrm{D}$. These and 
other values related with the physical dimensions and refractive power of the ICLs implanted, as well as the values of AC depth and limbus-to-limbus corneal diameter, are presented in Table 1. Mean objective and subjective vault values were $414 \pm 228 \mu \mathrm{m}$ and $2.1 \pm 1.0$, respectively. Comparison of means using Kruskal-Wallis test showed that there was a statistically significant difference in objective vault among different subjective vault groups $(P<.001)$ whose values are shown in detail in Table 2.

There was a statistically significant high correlation between subjective and objective values (Spearman rho; $r=.82 ; \mathrm{P}<.001)$. This correlation is graphically illustrated in the scatterplot of Figure 2. The frequency distribution of objective vault values among different subjective vault groups is best explored in Figure 3. Despite some overlap of same objective values for different subjective scales (side extremes of the Gaussian distribution), when the outliers are excluded, $99 \%$ of cases within the CI are in perfectly separated subjective groupings. Of course, this statistical calculation does not reflect totally what the surgeon can find in the clinic, but it is a good approach and is scientifically supported by statistical methodology.

Table 2 shows the descriptive statistics (mean, standard deviation, range, maximum and minimum) along with the 99\% CI lower and upper limits for objective vault measured with OCT corresponding to each grade of subjective vault. It is evident that within each value of subjective vault, $99 \%$ of eyes present an objective vault that is confined to a characteristic range that did not merge with objective values from other groups. According to this, in $99 \%$ of the eyes, each subjective grading value corresponds to an objective vault within an interval of $48 \mu \mathrm{m}$ for vault 0 , with $93.6 \%$ of them presenting vault below $100 \mu \mathrm{m} ; 54$ $\mu \mathrm{m}$ for vault 1 , with $92.8 \%$ of them presenting vault below $310 \mu \mathrm{m} ; 47 \mu \mathrm{m}$ for vault 2 , with $91.7 \%$ of them presenting vault beyond $620 \mu \mathrm{m} ; 79 \mu \mathrm{m}$ for vault 3 , with $73.0 \%$ of them presenting vault above $500 \mu \mathrm{m}$; and $162 \mu \mathrm{m}$ for vault 4 , with $81.0 \%$ of them presenting vault above $650 \mu \mathrm{m}$.

\section{DISCUSSION}

THE MAJOR CONCERN WITH PC IOLS INCLUDES ANTERIOR subcapsular cataract formation. In a study involving 75 myopic eyes undergoing ICL implantation, Gonvers and associates $^{15}$ evaluated central and peripheral vaulting of the ICL using a slit-lamp mounted camera and Scheimpflug photography. They showed that a central vault greater than $0.09 \mathrm{~mm}$ appears to protect crystalline lens from cataract formation and suggested a central vault greater than $0.15 \mathrm{~mm}$ as an ideal situation to avoid cataract formation after lens implantation.

Vault of an ICL is determined by its natural design curve and the relationship between ICL length and PC width. To improve the separation between the ICL and the anterior crystalline lens capsule and reduce the risk of touch between the 2 structures, the recent Visian ICL model, V4, was designed with higher intrinsic vault to avoid contact with crystalline lens capsule.

Despite the relevance of vault in clinical success of PC phakic IOLs, its measurement has been based essentially on subjective methods. Poor vault has been considered when the physical separation was inferior to $10 \%$ of the central corneal thickness (CCT) by some authors, ${ }^{16}$ while ideal vault is considered when a separation of about 150 $\mu \mathrm{m}$ or higher is present, which corresponds to about $30 \%$ of CCT. ${ }^{11}$ However, other methods have also been proposed. Zaldivar and associates ${ }^{6,19}$ used the following criteria: vault type 0 when there was no space between ICL and crystalline; vault type 1 when a small central space existed with a peripheral touch of crystalline; vault type 2 for a small space across the ICL diameter; and vault type 3 when an important space across entire ICL was present. Based on these criteria, a patient with a vault type 2 or 3 will have a lower potential to develop anterior subcapsular cataract. Elies and associates, ${ }^{18}$ using the same technique, classified vault as follows: negative vault, in which the ICL pressed and even depressed the front surface of the crystalline lens; vault 0 , when the ICL touches the anterior surface of the lens; vault 1 , where ICL was very close $(<100 \mu \mathrm{m})$ to the front of the lens, and there may exist peripheral contact between the lens and anterior lens capsule; vault 2 and vault 3, between $200 \mu \mathrm{m}$ and $450 \mu \mathrm{m}$ with central separation and no peripheral contact; vault $4(>500 \mu \mathrm{m})$, in which the ICL pushes the iris to the front of the eyeball. According to their experience vault 0 is associated with a very high-risk of cataract, which usually appeared within a few months, and vault 4 may be associated with a reduction of $\mathrm{AC}$ depth and a greater release of iris pigment and long-term significant damage and loss of corneal endothelial cells.

In the present study, objective vault measured following PC ICL implantation to correct myopia varied within a wide range of values, from 0 to $1300 \mu \mathrm{m}$ when measured objectively using OCT partial coherence tomography system. This wide range of values corresponded to subjective assessment values between 0 and 4 with almost half of the eyes (206 in 452) being graded subjectively as vault 2 , about $20 \%$ as vault 1 or 3 , and less than $10 \%$ as vault 0 or 4 in this setting.

With the exception of some few cases, subjective vault demonstrated to be a reliable way to assess and differentiate the separation between ICL and the natural lens of the eye. Except for vault 0 , an empirical relationship between subjective and objective vault values can be established. In this relationship, average objective vault within each group of subjective vault is obtained by multiplying the subjective grade by $200 \mu \mathrm{m}$; for $99 \%$ of the eyes the actual objective value will fall within \pm 25 to $\pm 80 \mu \mathrm{m}$ around this approximate value. For example, a subjective vault of 2 , which corresponds to the most common situation, will 
correspond in $99 \%$ of the cases within the $\mathrm{CI}$ to an actual value as measured with OCT of about $400 \mu \mathrm{m}$ within a range of $\pm 25 \mu \mathrm{m}$ above and below that value; similarly, for a subjective vault of 4 , the actual value will be about 800 $\mu \mathrm{m}$ on average within a range of $\pm 80 \mu \mathrm{m}$. Despite both examples agreeing with data presented in Table 2, this empirical rule of thumb should not be interpreted as a universal conversion relationship to derive actual values of lens-ICL separation from subjective vault values but to obtain an approximate average value.

However, clinical vault estimation comparing the physical separation between lens and ICL against CCT may lack objectivity because of simple fact that central thickness of normal corneas can vary within a wide range of values from 450 to $650 \mu \mathrm{m}$. To overcome this limitation, current technology allows for objective assessment to be made by a variety of new diagnostic tools such as ultrasound biomicroscopy (UBM), OCT, and Scheimpflug photography mounted in experimental or commercial devices. Choi and associates, ${ }^{24}$ using UBM, defined ideal vault between 250 and $750 \mu \mathrm{m}$, classifying excessive vault as values above $750 \mu \mathrm{m}$ and insufficient vault as values below $250 \mu \mathrm{m}$. Two studies using the P40 UBM in a series of 40 patients for clinical assessment of vault showed that all eyes identified as having a poor vault by clinical subjective estimation had poor vault measured objectively with UBM. ${ }^{1,2}$

Trindade and associates ${ }^{25}$ in their study using highfrequency UBM found contact between the iris and the ICL in all cases and contact between the crystalline lens and the ICL in 8 cases. Kim and associates ${ }^{26}$ used UBM to study 2 patients after ICL implantation and the depth of the lens vault varied from 34 to $214 \mu \mathrm{m}$; they also studied the influence of accommodation and change in pupil size, with the vault increasing during light-induced pupil constriction and near accommodation at a $30-\mathrm{cm}$ target and decreasing in non-accommodating conditions in total darkness. High-frequency UBM also has the capability to provide direct measures of relevant parameters in ICL surgery such as sulcus-to-sulcus distance, which is critical for accurate calculation of lens dimensions. ${ }^{24-26}$ However, this procedure requires a complex setup involving direct contact with the anterior ocular surface and also the use of topical anesthesia, with less potential for its application in a clinical surgical routine.

Optical coherence tomography technology is more widely used and provides an optical cross-sectional image of the anterior segment, allowing more precise evaluation of the anatomic relationship between the ICL and the crystalline lens in a noninvasive mode in a clinical setting. It also allows dynamic evaluation of changes in vault associated with accommodation and pupil size. ${ }^{21,22,27}$ Bechmann's study showed that lens vault changed from 136 to $438 \mu \mathrm{m}$, increasing with accommodation and decreasing under cycloplegia. ${ }^{22}$

Overall, we consider that subjective assessment, despite being clinically useful, may not preclude a surgeon's use of OCT or similar technology, when possible. Despite this, we consider that subjective evaluation is accurate enough to detect patients at a risk of having a cataract or increase in IOP related to surgical procedure. In our evaluations, the majority of patients with vault 0 as per subjective assessment have Visante values under $150 \mu \mathrm{m}$. Even when other authors ${ }^{15}$ have considered the number of $90 \mu \mathrm{m}$ $(0.09 \mathrm{~mm})$ as a cut-off point to consider risk for cataract development, all vault 0 should be considered for close follow-up. This recommendation could be extended to patients showing subjectively vault 1 because most of them will present Visante values below $250 \mu \mathrm{m}$, and considering some degree of forward shift of anterior lens surface with accommodation (more than $100 \mu \mathrm{m}$ ) this could place the patient at a higher risk of trauma to the lens.

On the other side of the scale, vault values subjectively assessed as 4 have Visante values above $600 \mu \mathrm{m}$, close to the $750 \mu \mathrm{m}$ considered excessive by other authors. ${ }^{24}$ This could place these patients at a higher risk of developing angle closure and subsequent acute rise in IOP, so they should be scheduled for tighter follow-up visits. Of course this recommendation should be extended to vault 4 .

In this study, we have shown that subjective values of vault assessed by a trained clinician are highly reliable in predicting an average value objectively measured using advanced technological devices. The evaluation of the CIs revealed that subjective vault can predict objective vault within a specific range of objective values measured with micrometer resolution. However, even when subjective assessment of vault can be adequate for clinical routine, objective methods should be used when possible. This is particularly important for research purposes as this method offers unique accuracy in the assessment of the physical separation between natural lens and implanted ICL for a better follow-up.

THIS STUDY WAS SUPPORTED IN PART BY A UNIVERSITAT DE VALENCIA RESEARCH GRANT NO. UV-AE-20070225\#, (DR Montés-Micó) and Fundação para a Ciência e Tecnologia of Portugal Grant no. FCT-SFRH-BD-34303-2007\# (Dr Fernandes). The authors indicate no financial conflict of interest. Involved in design and conduct of study (J.F.A., C.L., A.P., P.F., J.G.-M., R.M.-M.); collection, management, analysis, and interpretation of the data (J.F.A., C.L., A.P., P.F., J.G.-M., R.M.-M.); and preparation, review, or approval of the manuscript (J.F.A., C.L., P.F., J.G.-M., R.M.-M.). The study was approved by the Fernandez-Vega Ophthalmological Institutional Review Board. The study protocol was approved by and all study procedures adhered to the recommendations of the Declaration of Helsinki. 


\section{REFERENCES}

1. Sanders DR, Vukich JA, Doney K, Gaston M. U.S. Food and Drug Administration clinical trial of the implantable contact lens for moderate to high myopia. Ophthalmology 2003;110: 255-266.

2. Sanders DR, Doney K, Poco M. United States Food and Drug Administration clinical trial of the implantable collamer lens (ICL) for moderate to high myopia: three-year follow-up. Ophthalmology 2004;111:1683-1692.

3. Lackner B, Pieh S, Schmidinger G, et al. Long-term results of implantation of phakic posterior chamber intraocular lenses. J Cataract Refract Surg 2004;30:2269-2276.

4. Uusitalo RJ, Aine E, Sen NH, Laatikainen L. Implantable contact lens for high myopia. J Cataract Refract Surg 2002;28:29-36.

5. Alfonso JF, Palacios A, Montés-Micó R. Myopic phakic STAAR collamer posterior chamber intraocular lenses for keratoconus. J Refract Surg 2008;24:867-874.

6. Zaldivar R, Davidorf JM, Oscherow S. Posterior chamber phakic intraocular lens for myopia of -8 to -19 diopters. J Refract Surg 1998;14:294-305.

7. Pesando PM, Ghiringhello MP, Di MG, Fanton G. Posterior chamber phakic intraocular lens (ICL) for hyperopia: 10-year follow-up. J Cataract Refract Surg 2007;33:1579-1584.

8. Brandt JD, Mockovak ME, Chayet A. Pigmentary dispersion syndrome induced by a posterior chamber phakic refractive lens. Am J Ophthalmol 2001;131:260-263.

9. Bylsma SS, Zalta AH, Foley E, Osher RH. Phakic posterior chamber intraocular lens pupillary block. J Cataract Refract Surg 2002;28:2222-2228.

10. Sanchez-Galeana CA, Zadok D, Montes M, et al. Refractory intraocular pressure increase after phakic posterior chamber intraocular lens implantation. Am J Ophthalmol 2002;134: 121-123.

11. Chun YS, Park IK, Lee HI, et al. Iris and trabecular meshwork pigment changes after posterior chamber phakic intraocular lens implantation. J Cataract Refract Surg 2006; 32:1452-1458.

12. Trindade F, Pereira F. Cataract formation after posterior chamber phakic intraocular lens implantation. J Cataract Refract Surg 1998;24:1661-1663.

13. Arne JL, Lesueur LC. Phakic posterior chamber lenses for high myopia: functional and anatomical outcomes. J Cataract Refract Surg 2000;26:369-374.

14. Jimenez-Alfaro I, Benitez del Castillo JM, Garcia-Feijoo J, et al. Safety of posterior chamber phakic intraocular lenses for the correction of high myopia: anterior segment changes after posterior chamber phakic intraocular lens implantation. Ophthalmology 2001;108:90-99.

15. Gonvers M, Bornet C, Othenin-Girard P. Implantable contact lens for moderate to high myopia: relationship of vaulting to cataract formation. J Cataract Refract Surg 2003;29:918-924.

16. Sanders DR, Vukich JA. Incidence of lens opacities and clinically significant cataracts with the implantable contact lens: comparison of two lens designs. J Refract Surg 2002;18: 673-682.

17. Sanders DR. Anterior subcapsular opacities and cataracts 5 years after surgery in the visian implantable collamer lens FDA trial. J Refract Surg 2008;24:566-570.

18. Elies D, Coret A, Puig J, Rombouts A. Protocolos en el implante de lentes fáquicas tipo ICL. Annals d'Oftalmologia 2004;30-34.

19. Zaldivar R, Oscherow S, Piezzi V. Bioptics: Our personal experience. In: Probst LE, editor. LASIK: Advances, Controversies and Customs. Thorofare, New Jersey: SLACK Inc, 2003:249-257.

20. Baikoff G, Lutun E, Ferraz C, Wei J. Static and dynamic analysis of the anterior segment with optical coherence tomography. J Cataract Refract Surg 2004;30:1843-1850.

21. Baikoff G, Lutun E, Wei J, Ferraz C. Contact between 3 phakic intraocular lens models and the crystalline lens: an anterior chamber optical coherence tomography study. J Cataract Refract Surg 2004;30:2007-2012.

22. Bechmann M, Ullrich S, Thiel MJ, et al. Imaging of posterior chamber phakic intraocular lens by optical coherence tomography. J Cataract Refract Surg 2002;28:360-363.

23. Koivula A, Kugelberg M. Optical coherence tomography of the anterior segment in eyes with phakic refractive lenses. Ophthalmology 2007;114:2031-2037.

24. Choi KH, Chung SE, Chung TY, Chung ES. Ultrasound biomicroscopy for determining visian implantable contact lens length in phakic IOL implantation. J Refract Surg 2007;23:362-367.

25. Trindade F, Pereira F, Cronemberger S. Ultrasound biomicroscopic imaging of posterior chamber phakic intraocular lens. J Refract Surg 1998;14:497-503.

26. Kim DY, Reinstein DZ, Silverman RH, et al. Very high frequency ultrasound analysis of a new phakic posterior chamber intraocular lens in situ. Am J Ophthalmol 1998; 125:725-729.

27. Lege BA, Haigis W, Neuhann TF, Bauer MH. Age-related behavior of posterior chamber lenses in myopic phakic eyes during accommodation measured by anterior segment partial coherence interferometry. J Cataract Refract Surg 2006;32: 999-1006. 


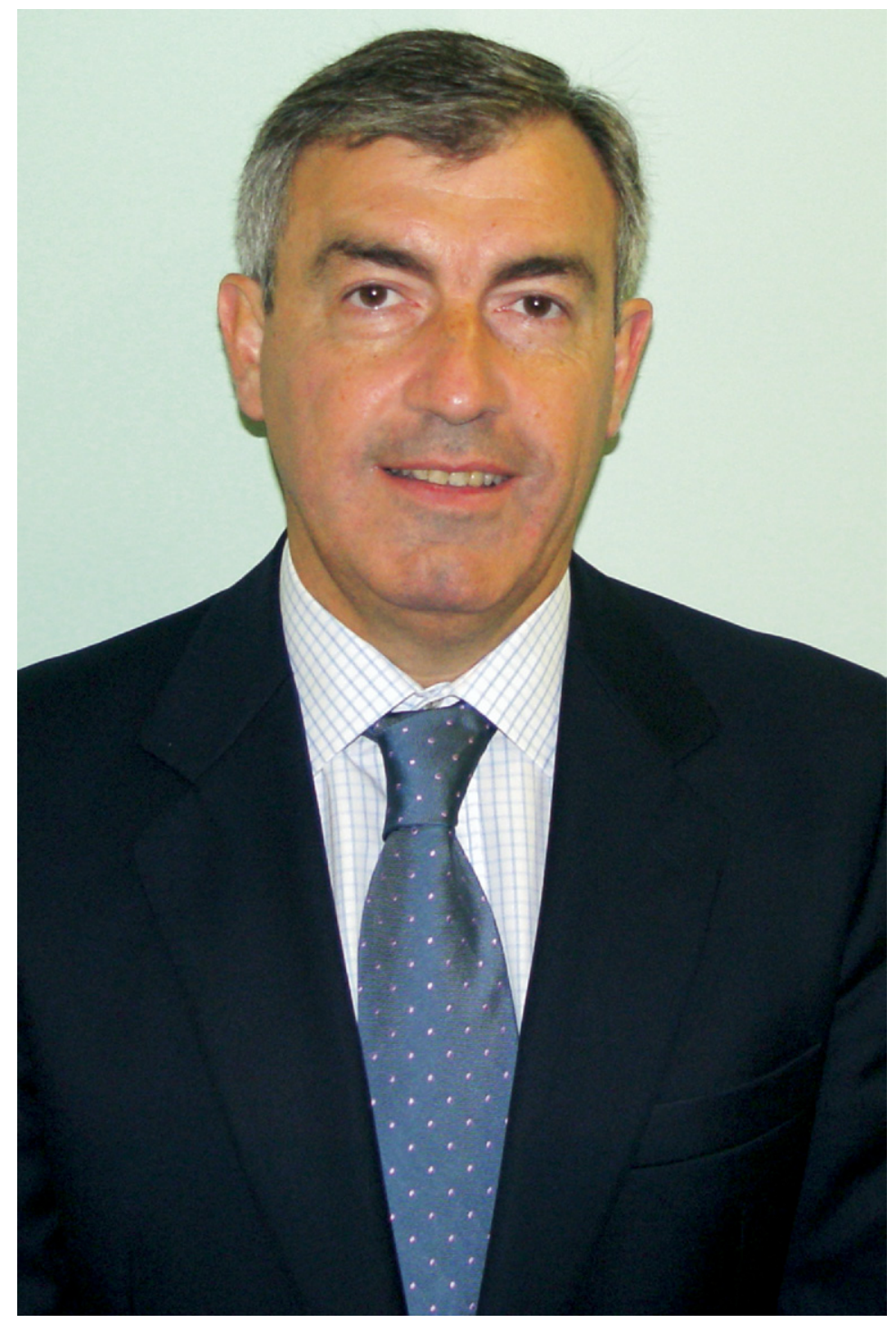

Biosketch

José F. Alfonso, $\mathrm{MD}, \mathrm{PhD}$, received his medical degree from the Complutense University of Madrid, Spain. After obtaining his MD, he performed his Residence in Ophthalmology at the Universitary Central Hospital of Asturias, Spain. He obtained his PhD in 1987 at the Complutense University of Madrid, Spain. Dr Alfonso is a Associate Professor at the University of Oviedo and Head of the Cataract and Refractive Surgery Section of the Fernández-Vega Opthalmological Insitute, Spain. 